\title{
Thermal Recovery of the NIF Amplifiers
}

S. Sutton, A. Erlandson, R. London,

K. Manes, C. Marshall, C. Petty,

R. Pierce, L. Smith, L. Zapata,

J. Beullier and B. Bicrel

This paper was prepared for submittal to the

Third Annual International Conference on Solid State Lasers for

Application (SSLA) to Inertial Confinement Fusion (ICF)

Monterey, California

June 7-12, 1998

June 30, 1998

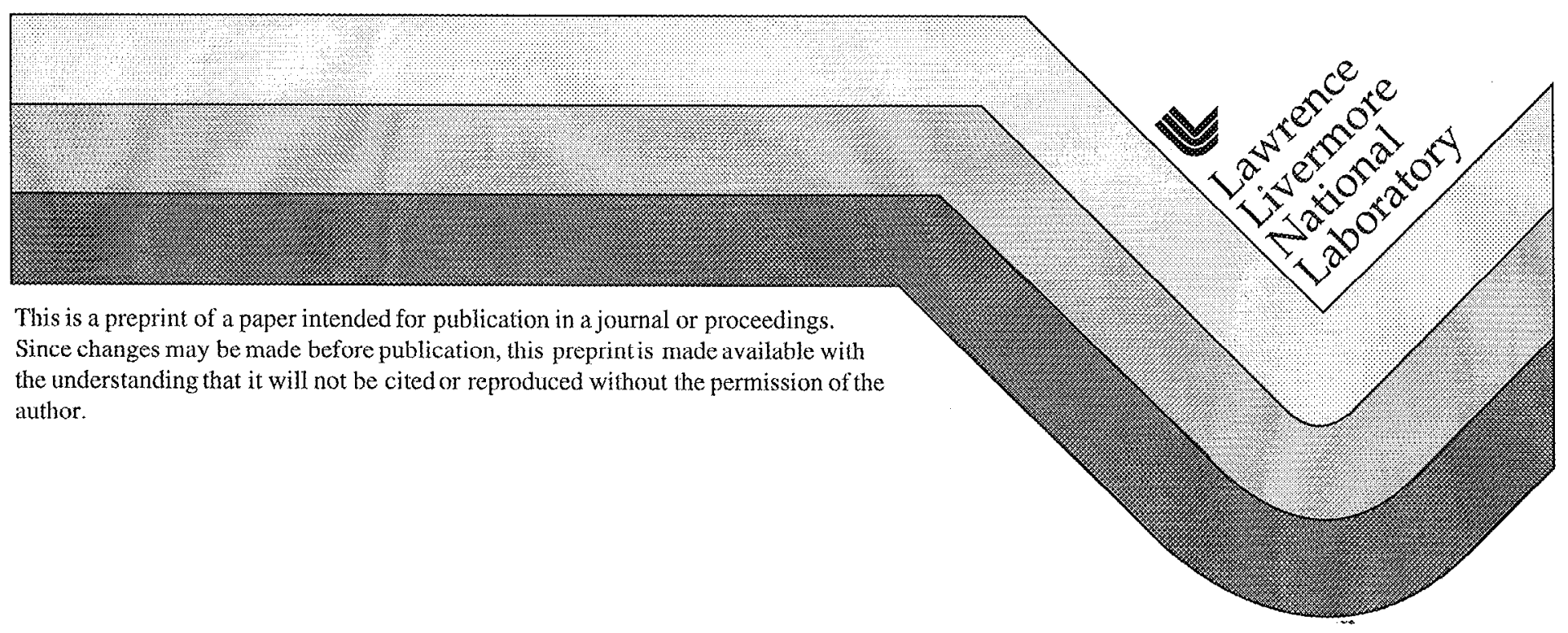




\section{DISCLAIMER}

This document was prepared as an account of work sponsored by an agency of the United States Government. Neither the United States Government nor the University of California nor any of their employees, makes any warranty, express or implied, or assumes any legal liability or responsibility for the accuracy, completeness, or usefulness of any information, apparatus, product, or process disclosed, or represents that its use would not infringe privately owned rights. Reference herein to any specific commercial product, process, or service by trade narne, trademark, manufacturer, or otherwise, does not necessarily constitute or imply its endorsement, recommendation, or favoring by the United States Government or the University of California. The views and opinions of authors expressed herein do not necessarily state or reflect those of the United States Government or the Universily of Califomia, and shall nol be used for advertising or product endorsement purposes. 


\title{
Thermal Recovery of the NIF Amplifiers
}

\author{
S. Sutton, A. Erlandson, R. London, K. Manes, C. Marshall, \\ C. Petty, R. Pierce, L. Smith, and L. Zapata \\ Lawrence Livermore National Laboratory \\ P.O. Box 808, L-441 \\ Livermore, CA 94550
}

J. Beullier and B. Bicrel

CEA Limeil-Valenton, DRTF/DI.P

94195 Villeneuve St-Georges, France

\begin{abstract}
With approximately $99 \%$ of the electrical energy supplied to the National Ignition Facility (NIF) appearing as heat in the amplifiers, thermal recovery of the NIF system is a major consideration in the design process. The NIF shot rate is one shot every 8 hours, with a goal of 4 hours between shots. This necessitates that thermal recovery take place in no more than 7 hours, with a goal of 3 hours for the accelerated shot rate. Residual optical distortions, which restrict the shot rate, are grouped into two discrete catcgorics: (1) distortions associated with residual temperature gradients in the laser slabs, and (2) distortions associated with buoyantly driven convective currents in the amplifier cavity and beam-tube regions. Thermal recovery of the amplifiers is achieved by cooling the flashlamps and blastshields with a turbulent gas flow. The cooled blastshields then serve as a cold boundary to radiatively extract the residual heat deposited in the slabs and edge claddings. Advanced concepts, such as the use of slightly chilled gas to accelerate some aspects of recovery, are addressed. To quantify recovery rates of the amplifiers, experiments and numerical models are used to measure and calculate the temperatures and optical distortions in NIF-like amplifier elements. The calculation results are benchmarked against AMLAB temperature measurements, thus allowing a quantitative prediction of NIF thermal recovery. These results indicate that the NIF requirement of 7 hour thermal recovery can be achieved with chilled temperature cooling gas. It is further shown that residual temperature gradient driven distortions in the slabs reach an acceptable level after 4 hours of thermal recovery.
\end{abstract}

Keywords: National Ignition Facility, laser amplifiers, thermal distortions, gas cooling, thermal recovery, numerical simulations, experiments

\section{INTRODUCTION}

The National Ignition Facility (NIF) laser will consist of 192 discrete apertures clustered into 2 wide $\times 4$ high bundles. Each 4-high aperture stack is separated by a flashlamp cassette creating isolated 1 wide $\times 4$ high cavities confined by sets of Brewster angle slabs. The beamtubes, which are attached to the ends of the 11-slab long power amplifier and 5-slab long booster amplifier are 2-apertures wide $x$ 4-apertures tall. Of concern in the design and development of the NIF amplifiers is removal of the waste heat, deposited in the system with each flashlamp firing, prior to the next shot. Current NIF requirements call for the system to fire once every 8 hours, with a goal of once every 4 hours. To accomplish this, the thermally driven optical distortions must reach an acceptably low level 7 hours after a shot in order to allow one hour of final alignment void of cooling system related disturbances. For the accelerated shot rate (once every 4 hours), the thermal recovery requirement becomes 3 hours. These requirements have necessitated the development of cooling approaches that efficiently and economically meet those recovery needs. 
We separate thermally related optical distortions in the NIF amplifiers and beamtube regions into two discrete categories: (1) optical distortions in the gas columns in the amplifiers cavities and beamtubes due to surface temperature difference driven convection flows, and (2) optical distortions in the laser slabs due to temperature gradients and their associated mechanical distortions. For thermally driven gas distortions, current requirements specify that distortions in the amplifiers and beamtubes shall not contribute more than an additional $5 \mu$ rad full-angle beam divergence. Prior analysis, using optical distortion measurements made on the Beamlet facility, relates this optical distortion limit to a $0.13^{\circ} \mathrm{C}$ allowable temperature imbalance in the system. ${ }^{1}$ For the NIF geometry this is interpreted to be the allowable temperature difference between the slab average temperature and the ambient temperature. For thermal distortions in the laser slabs, current requirements are less than 0.04 waves/slab/pass of optical distortion. Since these distortions add coherently, this translates to less than 2.2 waves total distortion for the NIF system (4-pass power amplifier and 2-pass booster amplifier).

Both requirements place severe restrictions on the allowable temperature imbalances in the system, and thus require the development of a cooling approach that meets or exceeds these design limits. This has necessitated a detailed experimental and modeling investigation of the time evolution of thermal energy in the amplifiers during the recovery process. Mock-up experiments were performed in the AMPLAB facility to measure temperatures in the slabs, blastshields, and flashlamps during the cooling cycle. Detailed numerical models of the NIF amplifiers were developed to calculate the temperatures and optical distortions during the recovery process.

In the remaining sections of this paper we describe the NIF cooling approach, the AMPLAB mock-up experiments, and the numerical models used to describe thermal recovery. We compare data to model results where applicable.

\section{NIF COOLING}

When considering cooling of any system, we should keep in mind that there are only three basic mechanisms available: conduction, convection, and radiation. In most average power laser systems, convective cooling using either a liquid or gas is the conventional approach. In these systems, the moving cooling fluid is in contact with both the laser medium as well as the excitation source (flashlamps or diodes). In NIF, which operates at a very low repetition rate and which structurally is very massive, an alternate approach was required. Recognizing that over $50 \%$ of the electrical energy supplied to the system appears as heat in the flashlamps, convective cooling of the flashlamps is an obvious requirement. Because of the large cavities and the requirement to maintain highly reflective solid surfaces throughout the system, direct convective cooling of the laser slabs is not feasible. Thus, an alternate mechanism is needed for reequilibrating the laser slabs. Radiative heat transfer is the mechanism of choice in this instance. The basic NIF geometry as well as the overall heat transfer approach is illustrated in Fig. 1. Each A-high stack of slabs is bounded on two sides by flashlamp cassettes. A blastshield lies between the lamps and slabs to provide protection to the slabs in the event of a lamp explosion. Convective cooling the flashlamp cassettes removes the waste heat from the flashlamps as well as maintains the blastshields as a cold barrier to radiatively extract heat from the slabs. The NIF baseline flashlamp cooling condition is $20 \mathrm{cfm}$ of ambient temperature nitrogen flowing over each lamp. To the vertically oriented lamps, which run the full length of the $1.8 \mathrm{~m}$ tall 4-high slab stack, this is provided as shown in Fig. 2. The lamp cassettes are arranged so that the flow is down one cassette, splitting and going up the neighboring cassettes.

Since the primary heat removal mechanism is convective cooling the flashlamp cassettes, recovery can be accelerated by slightly chilling the nitrogen cooling gas. Not only does this provided an enhanced removal rate of heat from the flashlamps and blastshields, but greatly improves the radiative coupling to the slab since the temperature difference between the blastshield and slabs is increased. Since the temperature difference between the slabs and blastshields is generally less than $0.5^{\circ} \mathrm{C}$, even a small reduction in the cooling gas temperature will greatly enhance the removal rate of waste heat from the laser slabs. This does require, however, that after some time the nitrogen cooling gas is returned to ambient temperature to ensure a satisfactory final temperature imbalance in the system. This chilled-gas enhanced recovery approach is addressed in detail later in this paper. 


\section{NUMERICAL MODELS}

Two numerical models have been used to quantify thermal recovery of the NIF amplifiers: (1) a lumpedmass model, and (2) a three-dimensional finite-element thermo-mechanical-optical model. In the lumpedmass model the amplifier cavity is grouped into five (5) major component categories with the masses and energies of each group lumped. The component groups are: slabs, side cassette lamps, side cassette blastshield, central cassette lamps, and finally central cassette blastshields. Radiative exchange factors are analytically derived based on the precise amplifier geometry. Analytically derived forced convection boundary conditions are applied to the flashlamps and blastshields. Included in this model are the effects of the down-up gas flow arrangement in pre-heating the cooling gas in $50 \%$ of the flashlamp cassettes. The overall purpose of this model is to predict the global temperature behavior of the system. Since the components are lumped, it is incapable of provided spatial information within a given component group.

The three-dimensional thermo-mechanical-optical model is used to study in geometric detail the thermal behavior of the NIF amplifiers. The multi-step calculation process starts with development of a finiteelement mesh that describes all solid components within the system. Included in the geometry are the reflectors, flashlamps, blastshields, slabs, and all relevant structural elements. The next step is calculation of radiation exchange factors for all surface elements in the system using a Monte-Carlo exchange factor code. $^{2}$ This is followed by evaluation of temperatures in the system for the entire recovery cycle using a finite-element heat transfer program. ${ }^{3}$ The fourth step is to extract temperature information for the slabs and edge claddings, and re-map it onto a mesh suitable for calculating thermal stresses and deformations. The finite element approach is then used to evaluate the stresses and deformations. ${ }^{4}$ The final step is to employ the calculated temperatures, stresses, and deformations in a ray-trace algorithm to calculate the optical path length changes across the aperture of a single slab. ${ }^{5}$ The thermal, mechanical, and optical properties of LG770 , as documented in Ref. 6, were used for the laser slabs. The properties of all other metal and glass components were typical values extracted from engineering handbooks.

\section{AMPLAB MEASUREMENTS}

A key aspect of this study was the instrumentation of AMPLAB to measure temperatures in the amplifier cavity. AMPLAB is a facility designed to test the optical and thermal performance of the NIF amplifiers. It consists of a NIF-like two slab wide configuration, but is only two slabs long in the optical propagation direction. The four slabs in each cassette are labeled A, B, C, and D from the top. One slab cassette, in what is termed the thermal measurement side (see Fig. 3) was instrumented with thermal measuring devices. Three slabs in the cassette (A, C, and D), the associated side cassette blastshield, and a side cassette flashlamp were similarly instrumented. A total of 59 type-E thermocouples and one fiber-optic probe were placed in the slabs. Typical locations of the thermocouples and fiber probe are shown in Fing. 4. The thermocouples were placed in $1 \mathrm{~mm}$ diameter holes drilled through the slab, with the junction at the midthickness of the slab. The fiber probe was placed in a blind hole drilled half-way through the slab. Four thermocouples were in contact with the flashlamp side of the blastshield, and four thermocouples and one fiber probe were in contact with one side cassette flashlamp. Thermocouples were selected (over thermisters) since they do not provide an added heat source which could alter the temperature reading in the low thermal conductivity glass slabs. It is estimated that the thermocouple accuracy is less than $\pm 0.05^{\circ} \mathrm{C}$. In some cases, the accuracy may have been as much as 5 times better. This instrumented cassette contained a combination of $34 \mathrm{~mm}$ thick and $40 \mathrm{~mm}$ thick slabs. All other slabs in the facility were $40 \mathrm{~mm}$ thick, which is the NIF thickness specification.

One key result of these measurements was quantification of starting conditions for use in simulations of both AMPLAB thermal recovery and NIF amplifier thermal recovery. The current starting condition set is given in Table 1, where flashlamp, edge cladding, blastshield, and slab temperatures are given for AMPLAB slabs, and NIF end and interior slabs. Several key features are noted. First, is the starting temperature differences for the AMPLAB $34 \mathrm{~mm}$ and $40 \mathrm{~mm}$ thick slabs. It was determined that simple thickness scaling is appropriate since the slabs are optically thick to the flashlamp light. That is to say, the temperature difference between the $34 \mathrm{~mm}$ and $40 \mathrm{~mm}$ thick slabs is $15 \%(40 \mathrm{~mm} / 34 \mathrm{~mm})$. Additionally, the interior slab temperature is $11 \%$ greater than the end slabs. Finally, the flashlamps in the AMPLAB thermal 
side cassette were judged to be $4^{\circ} \mathrm{C}$ hotter than the flashlamps in the optical side cassette because of differences in the reflector designs.

\section{MODEL AND MEASUREMENT COMPARISIONS}

In both numerical models, key system differences between AMPLAB and NIF were addressed. In the simulation of the thermal behavior of AMPLAB, there are fundamental differences between the "thermal" and "optical" sides of the system, as noted earlier. For instance, on the "optical" side all slabs are 40mm thick. On the thermal side, however, two slabs are $40 \mathrm{~mm}$ thick while the other two are $34 \mathrm{~mm}$ thick. In AMPLAB, all slabs are termed end slabs since the configuration is only two slabs deep. In NIF, where the amplifier will be at least 5 slabs deep, there are truly interior and end cassette geometries. These geometry variations were treated, as illustrated in Fig. 5, by capping off a single slab cassette by either symmetry planes (interior slabs) or an open end (end slabs). The open end was approximated as a perfect radiation absorber.

Since the flashlamps are the source of over $50 \%$ of the thermal energy in the system, it is imperative that the thermal models accurately model their cooling. Fig. 6 compares model results with thermocouple and fiber probe measurements. Because of prompt heating of both measuring devices, which requires several minutes to relax, the early-time temperatures are obtained from extrapolation. During most of this early stage of the recovery cycle, there is reasonably good agreement between model and measurement. Differences of as much as $15 \%$ are evident at times from 0.1 hour to 0.3 hours into recovery, and are likely attributable to uncertainties in the convection coefficient, the effect of the upstream cassette in pre-heating the cooling gas, and surface emissivities which control radiation transport. Differences of this magnitude, however, were no considered consequential in the overall recovery picture.

Optical distortions in the laser slabs are driven by temperature gradients both through the thickness and across the aperture. Since the edge cladding temperature rise is much larger than the bulk of the slab, the transport of energy to structural elements holding the slab is an important consideration in the models. The edge cladding region was investigated in detail to ascertain the effect of the slab mask and frame structure on the removal of waste heat. A simplified picture of the cassette frame and mask geometry, in relation to the slab and edge cladding, is given in Fig. 7. In this arrangement, besides conduction to the central regions of the slab, heat will also conduct through the thin air region between the slabs and masks. Based on numerical calculations, ${ }^{7}$ the projected starting temperature profile in the edge cladding is also shown in Fig. 7. In this instance, for the side edge claddings the peak temperature rise is $9.8^{\circ} \mathrm{C}$. A comparison of calculated and measured temperatures at the mid-thickness point of the edge cladding is given in Fig. 8. Note that excellent agreement is obtained using a conductance in the air-filled gap of $30 \mathrm{~W} / \mathrm{m}^{2}-\mathrm{K}$. This corresponds to conduction through a $1 \mathrm{~mm}$ thick gap, which is consistent with the assembled geometry. This knowledge of thermal behavior in the edge cladding region was incorporated into the detailed 3-D thermal model.

One key parameter in ascertaining thermal recovery is the average temperature rise of the laser slabs. After the early stages of recovery, the flashlamps and blastshields have been cooled to their initial ambient condition. Thus, the laser slab temperature represents the driving thermal potential for convective currents in the amplifier cavities and adjacent beamtubes. One check on model accuracy is to compare temperature predictions from the two independent thermal models. Fig. 9 compares the lumped-mass and 3-D model average slab temperature rise for both the interior slab geometry and the end slab geometry. Clearly evident is the remarkable agreement of the two models. It is noted once again that the lumped-mass model used analytically derived radiation exchange factors, while the 3-D model used a comprehensive Monte-Carlo exchange factor calculation algorithm. The excellent agreement between the two independent models serves to point out the accuracy of the exchange factor evaluation in the context of values used for surface reflectivities and enissivities.

Fig. 10 compares experimentally measured slab temperatures with their model counterparts. The experimental average slab temperature rise was determined by averaging the discrete thermocouple measurements that lied within the optical aperture of the slab. There is good agreement between model and experiment in the latter stages of recovery. However, clearly evident is the discrepancy in the first two 
hours of the recovery process where the model predicted temperature rise is as much as $20 \%$ greater than the experimental value. The origin of this discrepancy is unclear at this time and continues to be a focal point as we refine our understanding of the numerical models as well as the experimental results.

As addressed earlier, recovery can be accelerated by increasing the temperature differential over which the radiation exchange takes place. One way to accomplish this is to slightly reduce the temperature of the flashlamp cooling gas. Fig. 11 shows one such cooling profile, with the associated numerically calculated and experimentally measured slab temperatures given in Fig. 12. In this profile, the cooling fluid temperature is reduced by $0.5^{\circ} \mathrm{C}$ for the first 2.6 hours of the recovery cycle. It is then returned to ambient temperature for the remainder of the recovery process. Shown in Fig. 12 are model results for both ambient cooling and the chilled gas cooling cycle, along with the measured slab temperature. Again, agreement is excellent during the later stages of recovery, with the persistent nearly $20 \%$ difference again seen in the first 2 hours of the recovery cycle. Also evident is the benefit that this slight subcooling of the flashlamp cooling gas has on accelerating recovery. A mere $0.5^{\circ} \mathrm{C}$ temperature reduction results in a 2 hour decrease in the time it takes to drop the slab temperature to $0.1^{\circ} \mathrm{C}$ above ambient. This has strong implications for increasing the NIF shot rate with little additional facility expense.

\section{NIF PERFORMANCE PROJECTIONS}

Having established the utility of the numerical models to calculate temperatures in the NIF amplifiers during the recovery cycle, predictions of NIF thermal recovery can be made with some degree of confidence. First consider gas distortion related criteria, which as discussed earlier was that the slab temperature shall be reduced to less than $0.13^{\circ} \mathrm{C}$ above ambient within 7 hours after the start of recovery. Predictions for the thermal performance of the NIF amplifiers are given in Fig. 13. Results are presented for both end and interior slabs, and for both ambient cooling and chilled gas cooling. In the latter case, the flashlamp cooling gas temperature was reduced by $1^{\circ} \mathrm{C}$ for the first 2.5 hours of the recovery cycle. These calculations indicate that for the interior slabs, which start out at an $11 \%$ higher temperature than the end slabs, the derived criteria cannot be met in less than 8 hours. For the end slabs, which have a slightly lower starting temperature, the criteria can be met after 6.8 hours of recovery. However, by merely reducing the cooling fluid temperature by $l^{\circ} \mathrm{C}$, the recovery for both slab types can be reduced to 3 hours which meets the NIF accelerated shot rate requirements. This shows that only a slight sub-cooling would be required to meet the baseline 7 hour recovery condition for both interior and end slabs.

Thus far we have not addressed optical distortions in the laser slabs, which was the second recovery criteria mentioned earlier. These distortions are driven by temperature gradients in the laser slabs that contribute to both changes in the index of refraction of the laser medium as well as mechanical distortions in the slabs themselves. The process of evaluating the optical distortions was outlined earlier. A typical example of a distorted interior slab is shown in Fig. 14. In this case, the near side of the figure is facing the central flashlamp cassette. Because of modest cooling rate differences between the central and side cassettes, due to the geometry differences, the central cassette side is slightly cooler than the opposite side (which faces the side flashlamp cassette). This causes the warped shape shown in the figure. This mechanical distortion is a significant component of the optical path length variation across the aperture. A representative phase distortion through an interior slab is shown in Fig. 15. This is a tilt-corrected phase front, and is low order. Thus, it is amenable to deformable mirror correction. The calculated peak-to-valley optical path length variations for both interior and end slabs is given in Fig. 16. Again, this is tilt-corrected quantity. It is important to note that these current predictions indicate that the projected optical distortions are less than the NIF requirement which is 0.04 waves/slab/pass. To date we have been unable to extract suitable data from the AMPLAB experiments to validate these model results. We continue to refine the experiments as well as the numerical model predictions in an effort to ensure accuracy of our projections of amplifier slab optical distortions.

\section{SUMMARY AND CONCLUSIONS}

In this paper we have addressed thermal recovery of the NIF amplifiers with the goal of ensuring that the NIF design can achieve the desired 7 hour thermal recovery. This entailed detailed numerical predictions of 
the system temperature, measurements on AMPLAB of system temperatures, and numerical predictions of the slab optical distortions. In summary:

In addition to model predictions, temperature measurements were made in the AMPLAB facility for both ambient cooling gas and slightly chilled cooling gas. There was good agreement between the model results and the experimental measurements, which serves to validate the numerical models.

- Model results for the NIF amplifiers indicate that slightly chilled cooling as will be required to achieve 7-hour thermal recovery from the standpoint of gas optical disturbances. Results also indicate that only a $1^{\circ} \mathrm{C}$ temperature reduction is needed to achieve the 3 -hour accelerated shot rate recovery requirement.

- Numerical model predictions indicate that the slab residual thermal optical distortions will bo less than the NIF requirement for both 8-hour and 4-hour shot periods. Experimental results are not available at this time to validate these predictions.

\section{ACKNOWLEDGEMENTS}

This work was performed under the auspices of the U.S. Department of Energy by the Lawrence Livermore National Laboratory under contract no. W-7405-Eng-48. The authors wish to thank Buzz Pedrotti, Rick Sawicki and Steve Payne for their support of this work.

\section{REFERENCES}

1. S. Sutton, C. Marshall, C. Petty, L. Smith, B. van Wonterghem, and S. Mills, "Thermal recovery of the NIF amplifiers," Proceedings of SPIE: Second International Conference on Solid State Lasers for Application to Inertial Confinement Fusion, Vol 3047, pp. 560-570.

2. J. D. Maltby and P. J. Burns, "Users's Manual for MONTE2D and MONTE3D Monte Carlo Radiative Exchange Factor Codes," UCRL-MA-112192, February 1993.

3. A. B. Shapiro, "TOPAZ3D - A three-dimensional finite element heat transfer code," LLNL, UCID20484, August 1985.

4. B. N. Maker, et. al., "NIKE3D - A nonlinear, implicit, three-dimensional finite element code for solid and structural mechanics," LLNL, UCRL-MA-105268, Rev. 1, April 1995.

5. The optical propagation code used in this study is under continuing development at LLNL. The authors are indebted to W. Neumann and M. Rotter for providing access to this computer software.

6. J. H. Campbell, "Recent Advances in Phosphate Laser Glasses for High Power Applications," SPIE 1996 International Symposium on Optical Science, Engineering and Instrumentation, Denver, August $4-9,1996$.

7. Results of numerical simulations of edge cladding thermal loadings for NIF-like slabs as provided by J. Trenholme and K. Jancaitis. 
Table 1

Initial temperature conditions used in the AMPLAB and NIF thermal recovery calculations

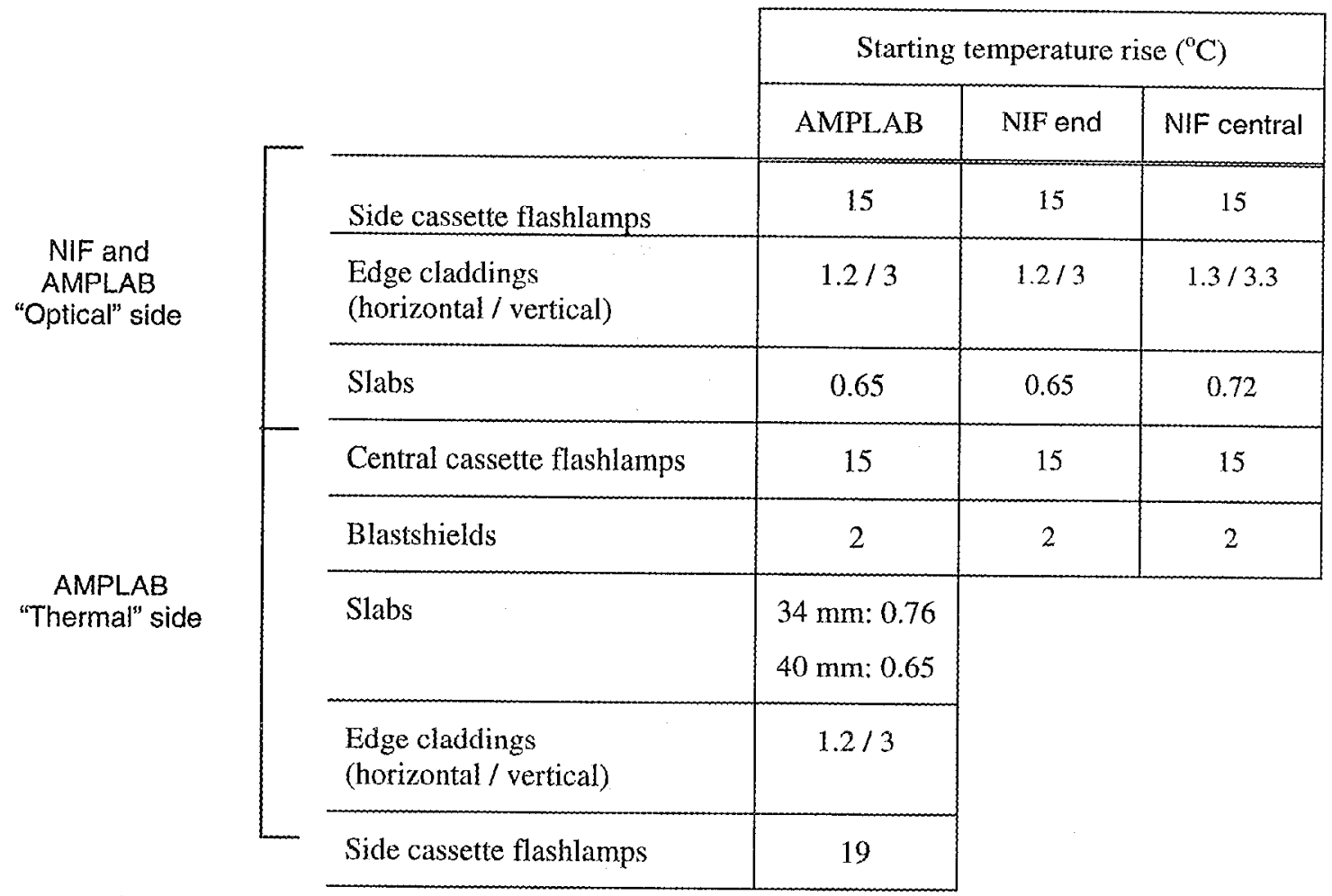

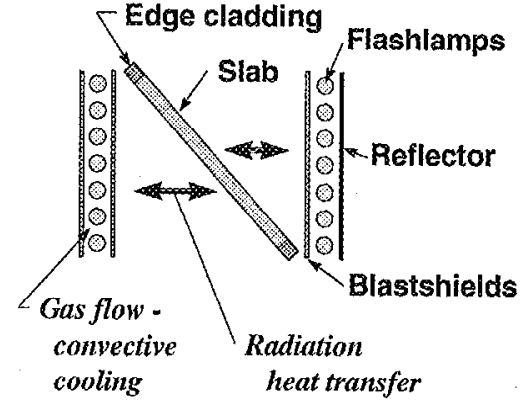

Fig. 1 - The major geometry elements and energy transfer mechanisms in the NIF amplifiers. Convective cooling removes heat from the lamps and blastshields. Radiative transport to the blastshields is the dominant mechanism for reequilibrating the slabs.

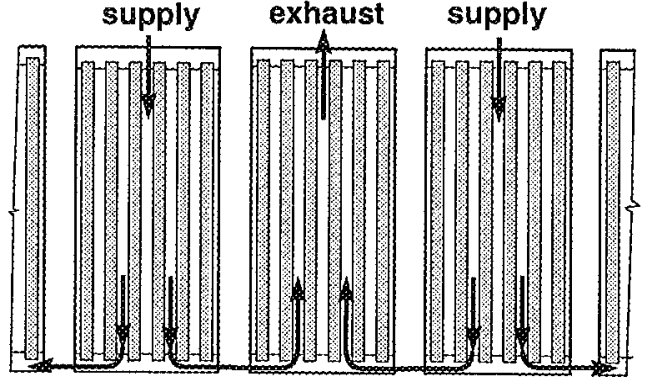

Fig. 2 - The flashlamp cooling flow configuration in NIF. The flow is in a down-up arrangement, with splitting to create a symmetric situation. 


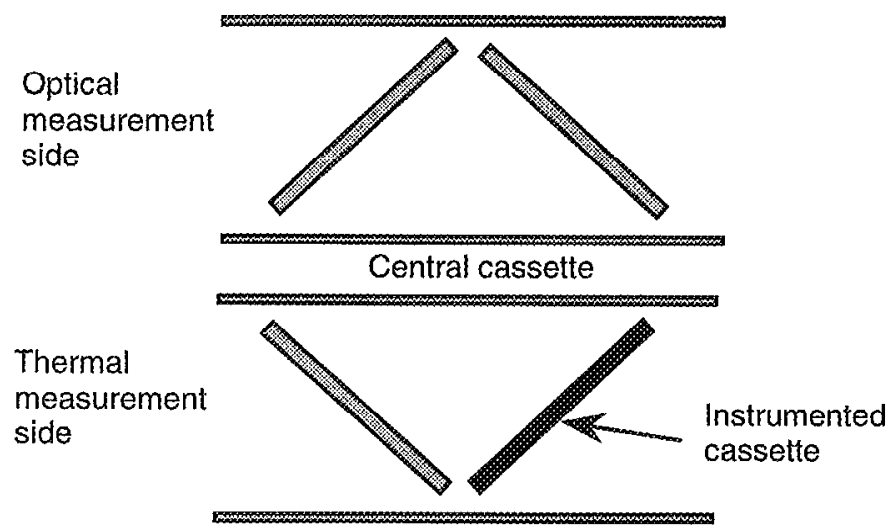

Fig. 3 - A plan-view schematic of the AMPLAB experimental configuration. One slab unit was instrumented with thermocouples to measure temperatures in the slabs during the thermal recovery cycle.

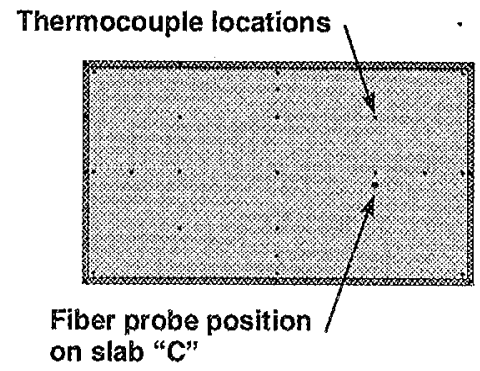

Fig. 4 - Typical locations of the thermocouples on slabs "A", "C", and "D", as well as the position of the fiber probe on slab "C"
Interior slabs: Mirror (symmetry) plane

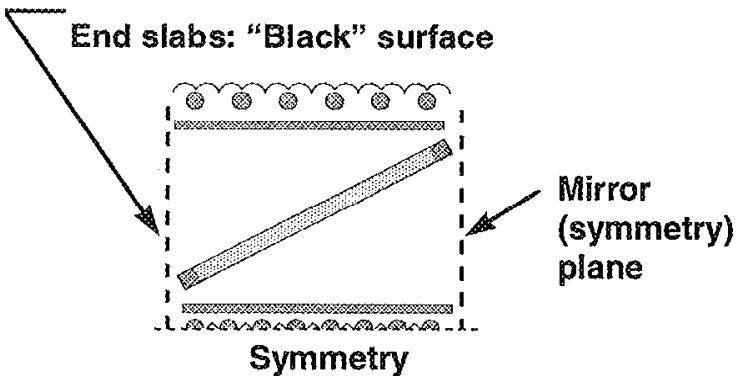

Fig. 5 - Description of boundary conditions used in the one-slab deep models to simulate interior and end slabs.

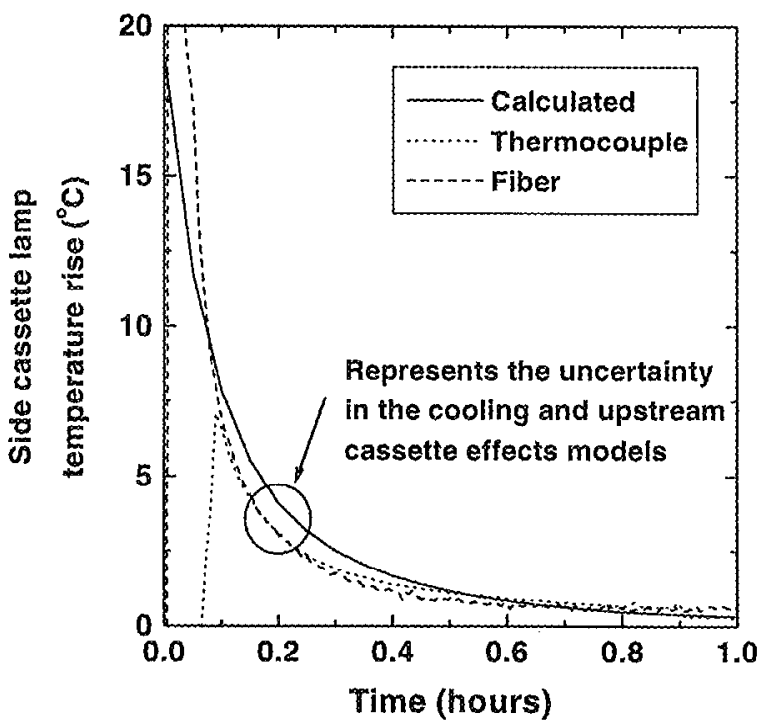

Fig. 6 - Comparison of the calculated and measured flashlamp temperature. Measured values using both thermocouples and the fiber probe are shown. 

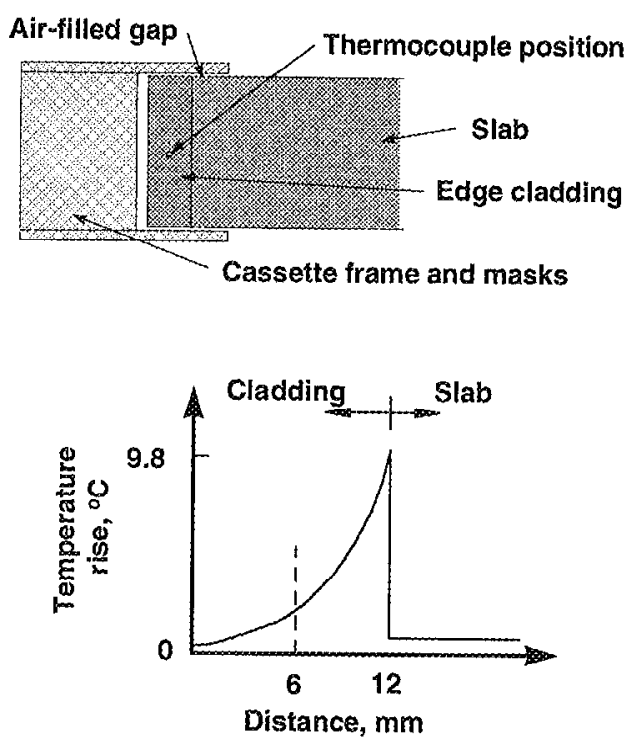

Fig. 7 - Simplitied representation of the slab frame geometry, and the temperature distribution used in the edge coupling analysis

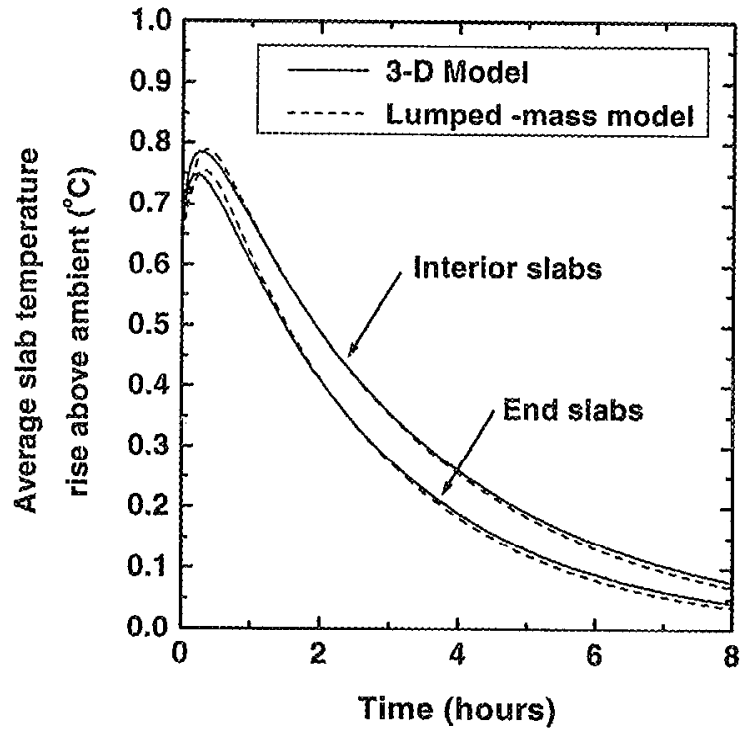

Fig. 9 - Comparison of the lumped mass and 3-D thermal models for recovery of both interior and end slabs.

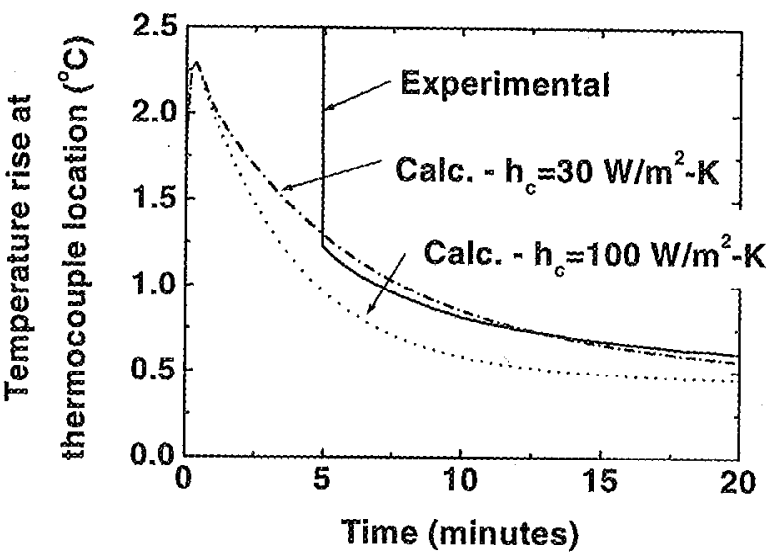

Fig. 8 - Comparison of calculated and measured temperature at the midpoint of the side edge cladding.

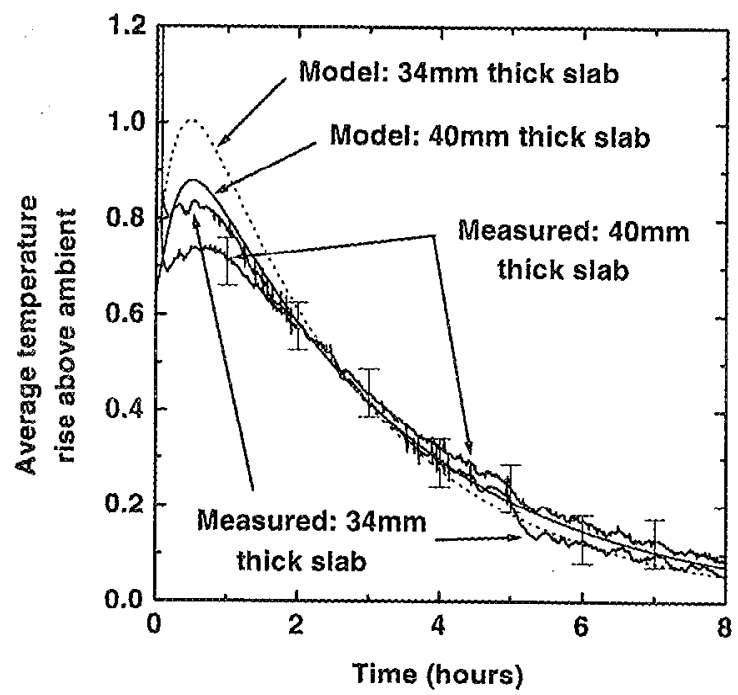

Fig. 10 - Comparison of measured and calculated average slab temperature rise for both the $34 \mathrm{~mm}$ and $40 \mathrm{~mm}$ thick slabs in AMPLAB. 


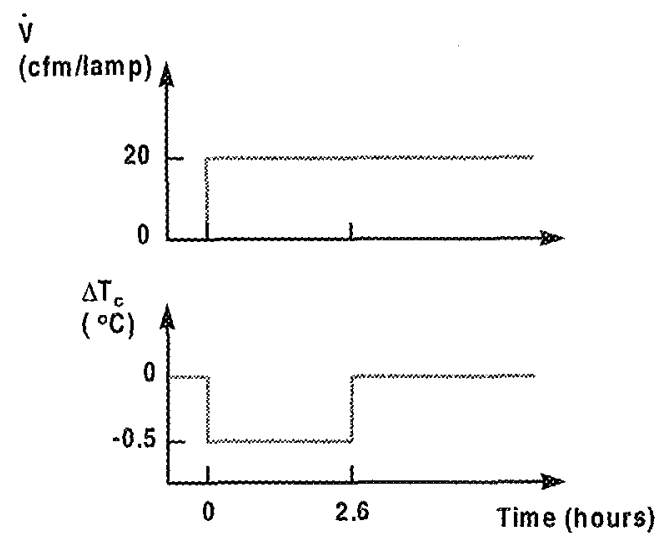

Fig. 11 - Temperature and flow velocity profiles used in the numerical simulations of chilled gas cooling. This is the nominal profile used in the AMPLAB experiment.

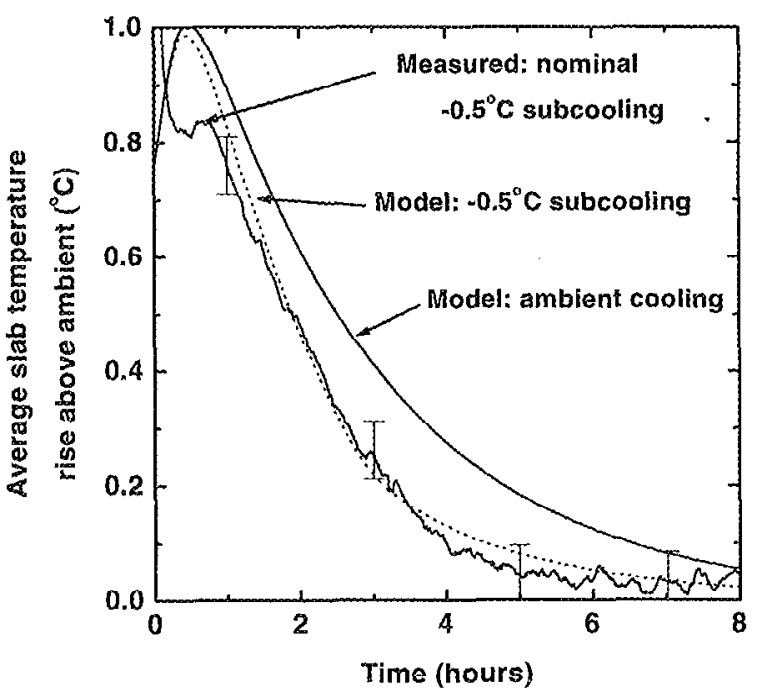

Fig. 12 -Comparison of calculated and measured average slab temperature rise for chilled gas cooling.

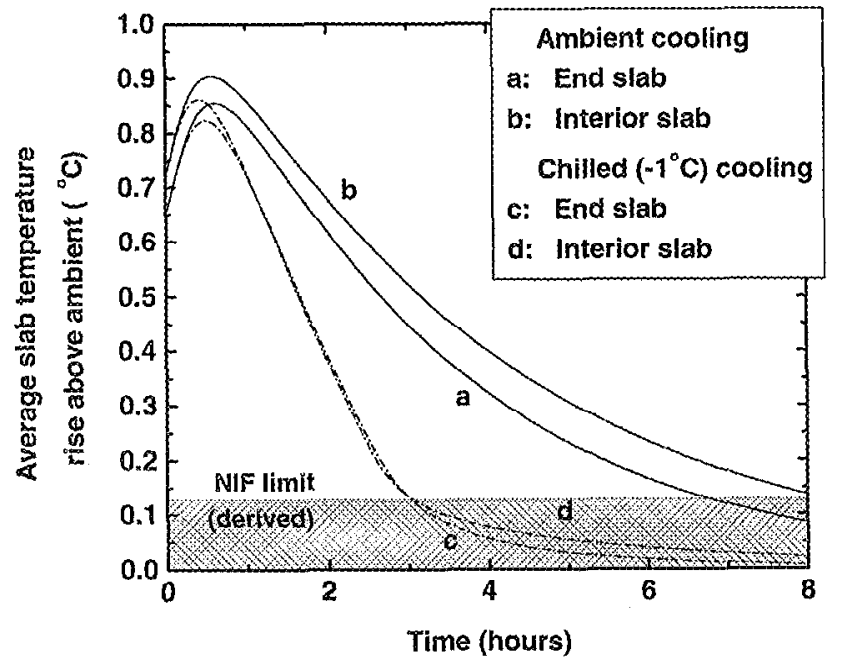

Fig. 13 - Calculated average slab temperature rise for NIF, for both ambient and chillcd gas cooling.

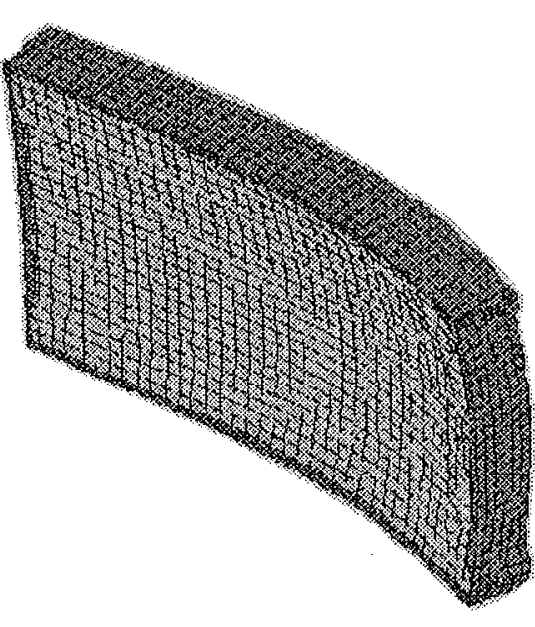

Fig. 14 - The deformed central slab as viewed from the central flashlamp cassette. Deformations are multiplied by $10^{6}$ in order to produce this image. 


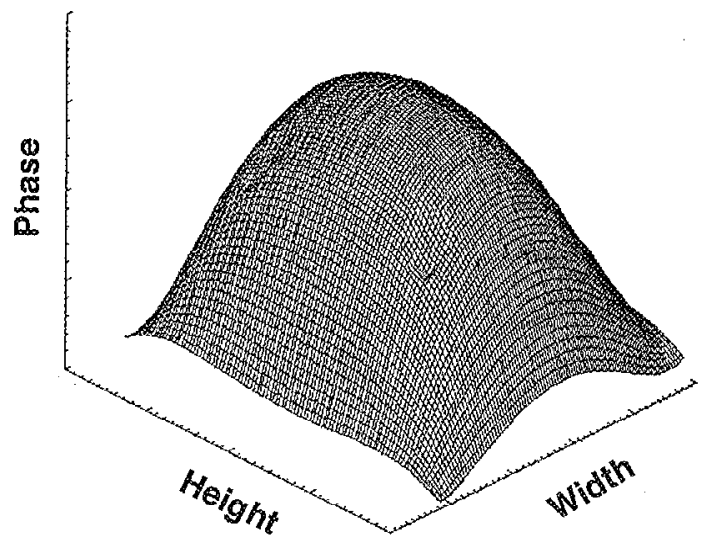

Fig. 15 - Representative distortion phase distribution resulting from temperature gradients and mechanical distortion of an interior slab.

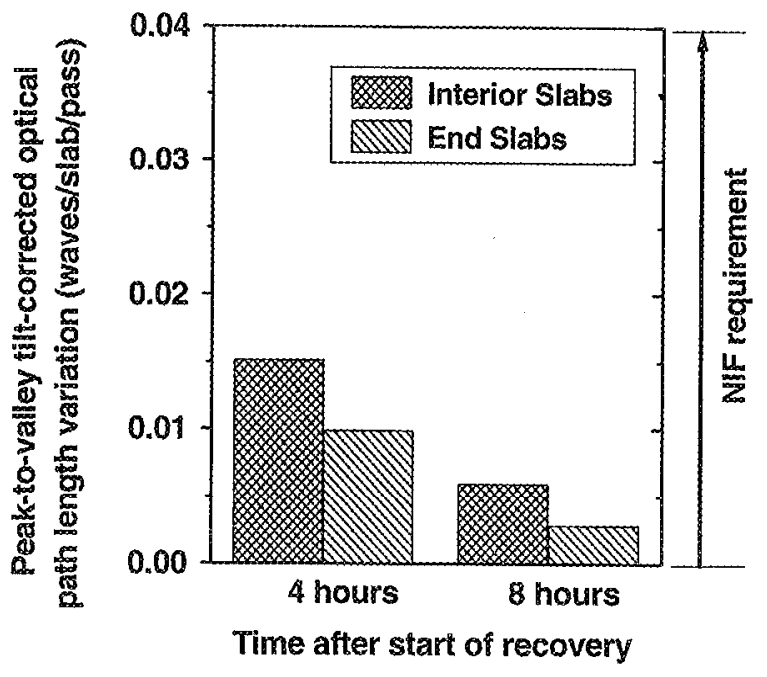

Fig. 16 - Calculated peak-to-valley optical distortions in the NIF amplifier slabs. 